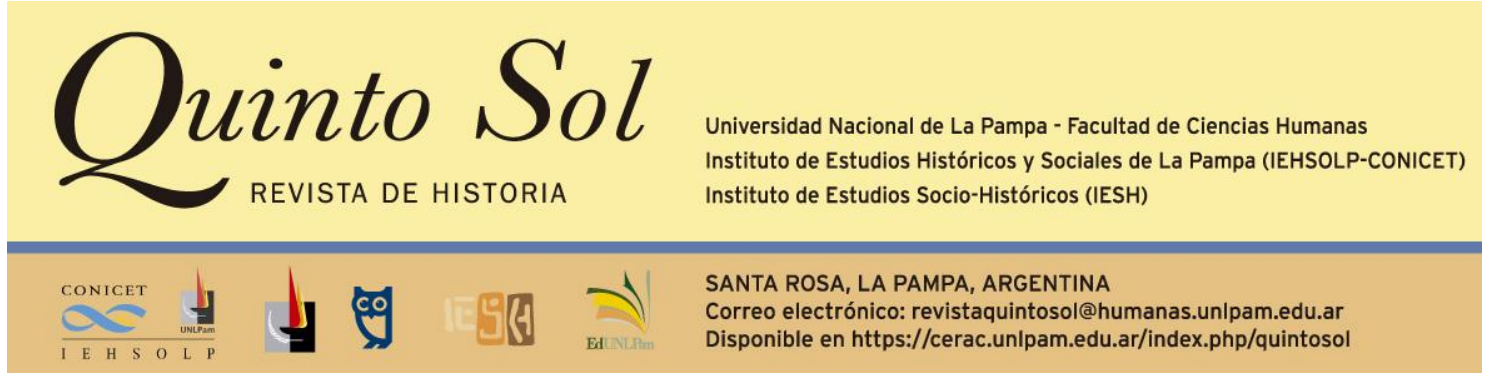

Quinto Sol, vol. 24, n 1, enero-abril 2020, ISSN 1851-2879, pp. 1-4

DOI: http://dx.doi.org/10.19137/qs.v24i1.3945

Esta obra se publica bajo licencia Creative Commons 4.0 Internacional. (Atribución-No ComercialCompartir Igual)

\title{
Cecilia Raffa e Ivana Hirschegger (Dirs.) Proyectos y concreciones. Obras y políticas públicas durante el primer peronismo en Mendoza (1946-1955). Mendoza: Universidad Nacional de Cuyo-Secretaría de Ciencia, Técnica y Posgrado, 2018, 217 páginas.
}

\author{
Eugenia Molina \\ Consejo Nacional de Investigaciones Científicas y Técnicas \\ Universidad Nacional de Cuyo \\ Argentina \\ Correo electrónico: eramolina@hotmail.com
}

La ampliación del campo de estudios sobre el peronismo ha permitido densificar las miradas sobre un fenómeno que ha resultado clave, qué duda cabe ya, para el proceso histórico argentino de la segunda mitad del siglo XX. El libro que aquí se reseña se integra en ese campo historiográfico, atendiendo a aspectos que, si en apariencia se vincularían solo con la urbanización y la arquitectura, dan cuenta, en cambio, de la fuerza ideológica que tuvo en sus distintas aristas un proyecto de país tal como el pergeñado por ese movimiento político. En tal sentido, la compilación aborda una serie de propuestas y acciones de obra pública en el marco del gobierno de Juan Domingo Perón, enfocadas en un espacio micro dado por la provincia de Mendoza, aunque aportando elementos interpretativos que pueden enriquecer los ejercicios comparativos con otras experiencias provinciales y la trayectoria de la gestión nacional. 
Con una excelente calidad de edición, lo que resulta fundamental dada la cantidad y diversidad del material gráfico incorporado a lo largo del texto, se inicia con un prólogo elaborado por la dra. Anahí Ballent, en el cual la especialista inserta este trabajo colectivo dentro de las discusiones actuales en torno de las relaciones entre estatalidad, técnica y política, como una línea de investigación que ha encontrado notable desarrollo en la última década en nuestro país, pero también a escala iberoamericana. A continuación, las directoras de la publicación introducen los vectores principales, presentando al lector los puntos nodales que han permitido articular sus secciones más allá de la especificidad temática abordada en cada una. En tal sentido, el primer capítulo, a cargo de Laura Ortega, se ocupa de la infraestructura vinculada a la gestión hídrica; en el segundo, Verónica Cremaschi realiza lo propio con la vivienda popular; en el tercero, Ivana Hirschegger aborda la problemática de la edificación conectada con la salud; en el siguiente, Isabel Durá Gúrpide reconstruye la proyectiva y elabora un balance acerca de las construcciones para educación; y en el séptimo, Cecilia Raffa da cuenta de los esfuerzos por "democratizar" el bienestar condensados en el concepto y acción del turismo social. Como cierre se incluye un último capítulo, también redactado por Raffa, que en tanto se dedica a la planificación de un centro cívico para el gobierno provincial, apuntando con ello al corazón y motor de la gestión local, cumple bien la función de completar una mirada de conjunto de los planes y los logros del período peronista respecto de la obra pública mendocina.

Aún con la amplitud de aspectos estudiados, se suma también la diversa formación disciplinar de sus autoras (que puede constatarse en los breves currículums incorporados al final), el libro logra una densa coherencia y una intensa articulación, esto expresa muy bien la noción de "proyecto" en un doble sentido. Por un lado, en vinculación con el trabajo en equipo de aquellas para acordar consensos teóricos y metodológicos más allá de sus objetos de análisis; y, por otro lado, en relación con la explicación e interpretación de lo que fue un esfuerzo de transformación del Estado en sus más distintos aspectos.

En relación con lo primero, esto es, con la idea de "proyecto" como una obra colectiva, se pueden detectar dos series de acuerdos que han permitido que esa referida diversidad aporte a una visión integral y compleja, y no genere, en cambio, una simple consecución de artículos cuyo único punto en común sea el signo partidario bajo el que se propusieron o realizaron las obras. Resulta clara, así, la existencia de un consenso teórico respecto de la adopción de una perspectiva del Estado desde adentro, es decir, desde la dinámica de las propias agencias que lo configuraron. En efecto, los distintos textos muestran cómo se fue construyendo desde lo material, lo simbólico e institucional el Estado de matiz peronista, reflejando que este calificativo no remite solo a una referencia partidaria sino a un amplio plan de transformación social. Una mirada tal les posibilita a las autoras dar cuenta tanto de las tensiones entre diferentes grupos corporativos y profesionales, como también del modo en que fueron variando las intensidades de proyección, ejecución y concreción según los resultados de las negociaciones resultantes entre ellos. Con este enfoque, entonces, logran fortalecer las argumentaciones respecto de la porosidad y heterogeneidad estatal en la línea de lo que viene planteando el campo de estudios conformado en torno de sus burocracias y agencias.

No obstante, otro consenso, digamos, metodológico, se advierte en el denso cruce de fuentes que sostiene las exposiciones. De tal forma, si en todos los capítulos 
se apela a documentos gubernamentales (tanto del Poder Ejecutivo como del Legislativo), también se utilizan los repositorios institucionales producidos por las mismas oficinas conectadas con los aspectos sociales analizados (por ejemplo, el Departamento General de Irrigación o el Instituto Provincial de la Vivienda). En este aspecto, la opción de mirar al Estado desde adentro se evidencia una vez más al develar en qué medida el discurso estatalista alimentado por sus propias agencias legitima su penetración social tanto como lo hace respecto de estas mismas en el concierto (y negociación) de poderes. A ello se suma la incorporación general de fuentes periodísticas y editoriales, esta inclusión contribuye a restituir el espacio de experiencia desde el cual se plantearon los proyectos y el horizonte de expectativas al cual apuntaban.

Sin embargo, junto a esas elecciones teórico-metodológicas, un conjunto de ejes analíticos también brindaría coherencia al trabajo colectivo. Se pueden detectar, de hecho, al menos cinco tríos/pares conceptuales en tensión que todos los capítulos articulan desde su particular foco de interés. Uno de ellos tiene que ver con la relación entre municipio-provincia-nación, la cual intentan reconstruir para dar cuenta de los márgenes de autonomía que las autoridades locales de diferentes niveles tuvieron frente a la supuesta centralización que el peronismo habría impuesto, según ciertas tesis consagradas. El juego interpretativo entre continuidad y ruptura, a su vez, conforma otro elemento que atraviesa los textos compilados, esto resulta clave para la renovación de la mirada sobre los vínculos del peronismo con los gobiernos anteriores (lencinistas y conservadores), cuya gestión de la obra pública parece haber sido fundamental para consolidar elencos de expertos y ejercitar proyectivas en experiencias previas, una cuestión que permitiría explicar la posterior concreción de ciertas obras durante el período 1946-1955. Por su parte, la reconstrucción de la bipolaridad centroperiferia en cada caso, les permite ofrecer una perspectiva compleja de la territorialidad provincial, en la cual el equipamiento político-institucional y el financiamiento se develan como decisiones de jerarquización para nada neutras y objetivas. La relación entre técnica y gobierno, además, demuestra (del mismo modo que recientes estudios para otros contextos espaciales y temporales) la intensidad de su lazo dialéctico, por el cual, si los saberes y experticias lograron la legitimación del Estado y protagonismo en sus acciones, este último se sirvió de aquellos para ampliar y profundizar su intervención en la sociedad. Finalmente, la dinámica entre los conceptos de proyecto y concreción (que da título a la obra), conforma un recurso analítico central para la cohesión interna de ésta. Así, todos los textos incluyen los planes propuestos, restituyendo el valor que las aspiraciones jugaron al configurar un horizonte de llegada, como sus efectivas ejecuciones, ofreciendo completos balances de los aspectos tratados que permiten poner en discusión en clave comparativa conclusiones obtenidas para otros casos provinciales y/o el nacional.

Ahora, retomando ese referido segundo sentido con el cual el libro reflejaría, en nuestra opinión, la noción de "proyecto", es claro cómo da cuenta detallada y explícita de un programa de transformaciones que debían afectar a la sociedad en sus más disímiles aspectos, esto quizá permita pensar la posibilidad de un Estado "peronista". De tal forma, los textos reunidos demuestran las articulaciones entre las diferentes dimensiones (infraestructurales, institucionales y simbólicas) de la estatalidad, evidenciando la voluntad de un proyecto ideológico que intentaba reformarla en todos (o al menos muchos) de sus frentes. En este sentido, una de las virtudes de esta obra 
colectiva es que logra reflejar las dinámicas relacionales sectoriales dentro de un amplio programa de políticas públicas que integraban educación, salud y vivienda como un trípode basal, que se conectaba también con un esfuerzo por democratizar el ocio, amplificar la potencialidad del agua como recurso vital en territorios de aridez generalizada, y dotar al gobierno de un lugar material de publicidad representativa. En definitiva, las autoras muestran cómo la proyectiva pública del período analizado se sirvió de experiencias previas, de elencos de expertos de creciente consolidación y del fortalecimiento de ciertas estructuras burocráticas para producir un discurso y una acción legitimadora de su propia gestión, alimentando lo que quizá podría llegar a plantearse como una ampliación y profundización de la estatalidad con una modalidad política específicamente peronista.

Para concluir, conviene llamar la atención sobre un detalle nada menor respecto del formato de edición elegido para este libro: una publicación digital de libre acceso alojada en el repositorio de la Universidad Nacional de Cuyo (bdigital.uncu.edu.ar/11476). Al respecto, creemos que contribuirá seguramente a una mayor circulación y lectura crítica del texto, y nutrirá el debate de los dos campos historiográficos ya referidos con cuyos problemas dialoga: el de los estudios del peronismo, por un lado, y el de la configuración de las agencias estatales, por el otro. 